\title{
Analysis of Frankia populations in three soils devoid of actinorhizal plants
}

\author{
Liisa Maunuksela ${ }^{\mathrm{a}, *}$, Kornelia Zepp ${ }^{\mathrm{b}}$, Teija Koivula ${ }^{\mathrm{a}}$, Josef Zeyer ${ }^{\mathrm{b}}$, \\ Kielo Haahtela ${ }^{\mathrm{a}}$, Dittmar Hahn ${ }^{\mathrm{b}}$ \\ ${ }^{a}$ Department of Biosciences, Division of General Microbiology, University of Helsinki, P.O. Box 56 (Viikinkaari 9), \\ FIN-00014 Helsinki, Finland \\ ${ }^{\mathrm{b}}$ Federal Institute of Technology (ETH Zürich), Institute of Terrestrial Ecology, Soil Biology, Grabenstr. 3, 8952 Schlieren, Switzerland
}

Received 20 April 1998; received in revised form 17 August 1998; accepted 4 September 1998

\begin{abstract}
Frankia populations were analyzed in three soils devoid of actinorhizal plants but containing monocultures of birch (Betula pendula Roth), pine (Pinus sylvestris L.) or spruce (Picea abies (L.) Karsten). Bioassays using seedlings of Alnus incana as capture plants resulted in nodulation capacities of $3160 \pm 7,2267 \pm 13$, and $2747 \pm 6$ nodulation units $\mathrm{g}^{-1}$ of these soils, respectively. Comparative sequence analysis of an actinomycetes-specific insertion in domain III of the 23S rRNA allowed a grouping of isolates obtained from nodules of the capture plants into three distinct groups of the Alnus host infection group. This separation was confirmed by the analysis of genomic fingerprints of the isolates generated by rep-PCR fingerprinting with the BOX primer. Genomic fingerprints also demonstrated that all isolates differed from each other. The isolates accounted for a significant proportion of the Frankia population in root nodules of the capture plants as shown by in situ hybridization with specific probes. However, only those Frankia strains isolated from soil of the birch stand via Alnus seemed to represent the total Frankia population in root nodules. Nodules induced after inoculation with soil from the pine or spruce stand also contained Frankia populations which were not isolated during this study and which could not be identified by in situ hybridization. Depending upon whether the soil originated from a birch, pine or spruce stand, different Frankia populations were found in the nodules of the capture plants. Because a nested PCR on nucleic acids extracted from these different soils did not indicate differences in the diversity of the total Frankia populations, it was concluded that Frankia populations in nodules of the capture plants represent the fraction of physiologically active, infecting frankiae in the soils rather than the total Frankia population. (C) 1999 Federation of European Microbiological Societies. Published by Elsevier Science B.V. All rights reserved.
\end{abstract}

Keywords: Frankia; Alnus incana; Nodulation capacity; Ribosomal RNA; In situ hybridization

\footnotetext{
* Corresponding author. Tel.: +358 (9) 70859 204; Fax: +358 (9) 70859 262; E-mail: liisa.maunuksela@helsinki.fi
}

\section{Introduction}

Studies on the ecology of natural populations of Frankia are largely impeded by difficulties in isolating and growing these organisms [1,2]. In fact, only 
one successful isolation of Frankia directly from soil has been reported [3]. More commonly, Frankia isolates are obtained from root nodules, which serve as a natural enrichment for this organism [4]. Different isolation procedures have successfully been used during the last years, and hundreds of isolates are available (as reviewed in [5]). However, all isolates belong to the spore $(-)$ type, whereas isolates producing spores in root nodules (spore (+) type) have not been obtained in pure culture yet. Even for Frankia strains of the spore (-) type, so far no general isolation protocols have been developed and consequently only small percentages of isolation attempts succeed $[6,7]$.

Quantitative studies on Frankia populations are usually done by plant bioassays in which a quantification of the nodulation capacity on a specific host plant is used to describe the infective Frankia population (expressed as nodulation units $\mathrm{g}^{-1}$ soil) [8]. This approach has been combined with the concept of most probable numbers (MPN) in which host plants inoculated with serial dilutions of Frankia containing samples are only analyzed for qualitative nodule formation $[9,10]$. By these methods, nodulation units between 0 and $4600 \mathrm{~g}^{-1}$ soil have been obtained for different soils [11]. These results demonstrate that members of the genus Frankia can survive and remain infective in soils that are devoid of host plants [12-17]. The nodulation capacity of soil from birch stands, for example, appears to be as high as or higher than that of soil from alder stands [16$18]$.

However, apart from the need for vast numbers of test plants, this bioassay is hampered by its extreme selectivity because only nodule-forming Frankia populations can be analyzed. Furthermore, this bioassay is not feasible for addressing questions on Frankia populations belonging to host infection groups other than the test plants [19], on nonnodulating Frankia populations of the same host infection group [20,21], on competition for infection between Frankia populations in one sample, and on variable compatibilities of host plants to different Frankia populations [21-24]. Furthermore, the correlation of the nodulation unit, which can theoretically be induced by a single spore, a hyphal fragment or a colony, with cell numbers remains difficult [11].
The aim of our study was to analyze Frankia populations in three soils devoid of actinorhizal plants but containing monocultures of birch (Betula pendula Roth), pine (Pinus sylvestris L.) or spruce (Picea abies (L.) Karsten). Major emphasis was laid on the comparative analysis of uncultured Frankia populations in soils and those populations in root nodules obtained on Alnus incana seedlings that were used as capture plants in bioassays. An actinomycetes-specific insertion in domain III of the $23 \mathrm{~S}$ rRNA served as a specific marker for different Frankia populations [25]. These populations were analyzed by molecular detection methods such as the polymerase chain reaction (PCR) and subsequent probing [26] or by the in situ hybridization technique $[26,27]$.

\section{Materials and methods}

\subsection{Soil characteristics}

Surface samples (down to $10 \mathrm{~cm}$ depth) were taken from block number 2 of an afforestation site ( 0.2 ha) at Karttula ( $62^{\circ} 52^{\prime}$ N, $27^{\circ} 10^{\prime}$ E, $98 \mathrm{~m}$ asl), Finland, containing monocultures of birch (Betula pendula Roth), pine (Pinus sylvestris L.) and spruce (Picea abies (L.) Karsten) which were planted in 1970 after hay had been grown at the site for many years [28]. The characteristics of these three stands were similar with respect to basic physical and chemical parameters, essentially comprising a mineral soil with the soil texture class of silty fine sand containing 12 $14 \%$ organic material at a $\mathrm{pH}$ between 5.3 and 5.5 [28]. A more detailed description of the characteristics of both the mineral soil and the organic material of the three sites and of the site history is given in $[28]$.

\subsection{Analysis of nodulation capacity}

Alnus incana (L.) Moench was used as a capture plant in a bioassay to analyze the nodulation capacity of soils from birch, pine or spruce stands. Surface-sterilized seeds were germinated and grown axenically in glass bottles supplemented with halfstrength nutrient solution at a $\mathrm{pH}$ of 5.8 [16]. Three replicate bottles containing 5-week-old seed- 
lings were inoculated with 20 -fold dilutions of this soil suspension after dispersion of $5 \mathrm{~g}$ of soil in $50 \mathrm{ml}$ of low-N nutrient solution by repeated shaking, so that the amounts of soil per bottle equalled $0.5 \mathrm{~g}, 0.025 \mathrm{~g}$ and $0.00125 \mathrm{~g}$ [16]. After 5 weeks of growth under conditions described in [16], plants were analyzed for root nodule formation. Number of nodules was expressed as nodulation units $\mathrm{g}^{-1}$ soil [8].

\subsection{Isolation and cultivation of Frankia}

For the isolation of frankiae, about half of the nodules obtained on $24 \mathrm{~A}$. incana seedlings were surface-sterilized with $1 \%(\mathrm{w} / \mathrm{v}) \mathrm{OsO}_{4}$ for $1-3 \mathrm{~min}$, washed with distilled water, and subsequently cut into small pieces [29]. Per nodule, 4-33 tubes containing either TPC+glucose medium, TPC medium or PC medium [30] were inoculated and incubated at $28^{\circ} \mathrm{C}$. Subcultures were grown in TPC or PC medium. Isolates obtained were encoded with acronyms (AiBp1-6 for isolates 1-6 from A. incana inoculated with soil from the $B$. pendula stand, AiPs1-4 for isolates 1-4 from $A$. incana inoculated with soil from the $P$. sylvestris stand, and AiPal for the isolate from $A$. incana inoculated with soil from the $P$. abies stand).

\subsection{Nucleic acid extraction}

Nucleic acids from isolates were extracted after enzymatic lysis with lysozyme $\left(10 \mathrm{mg} \mathrm{ml}^{-1}\right.$, Fluka) at $37^{\circ} \mathrm{C}$ for $1 \mathrm{~h}$, subsequent incubation with proteinase $\mathrm{K}\left(1 \mathrm{mg} \mathrm{ml}{ }^{-1}\right.$, Appligene) in the presence of $0.1 \%$ (v/v) $\mathrm{SDS}$ at $37^{\circ} \mathrm{C}$ for $45 \mathrm{~min}$ and final addition of SDS to $2 \%$ and incubation at $65^{\circ} \mathrm{C}$ for $15 \mathrm{~min}$. Nucleic acids were purified with phenol/chloroform extraction, precipitated with ethanol, dried and resuspended in distilled water [31].

Nucleic acids were obtained from soil samples after cell lysis by a modified bead beating protocol [32]. Released nucleic acids were extracted with phenol/chloroform, precipitated with ethanol, dried and resuspended in $40 \mu \mathrm{l}$ of distilled water [31]. The nucleic acids were further purified on Sephadex G-200 columns [33], again extracted with phenol/chloroform, precipitated, dried and resuspended in $100 \mu \mathrm{l}$ of distilled water.

\subsection{Nucleic acid sequencing and sequence analysis}

For sequence analysis, the actinomycetes-specific insertion in domain III of the 23S rRNA of the isolates was amplified by PCR. As PCR primers, oligonucleotides 23InsV and 23InsR [34] with $5^{\prime}$ end extensions of M13 reverse (5'-TTT CAC ACA GGA AAC AGC TAT GAC-3') and universal (5'-CGA CGT TGT AAA ACG ACG GCCA-3') primers were used to facilitate the subsequent sequencing. Primers used in sequencing were fluorescein-labeled M13-RP primer for one strand and 23Fra [25] for the complementary strand. The sequencing of PCR amplification products was carried out by the cyclic dideoxy method using thermoSequenase (Amersham) and sequencing reactions were analyzed using an automated ALF DNA sequencer (Pharmacia). Sequence analysis was done with the Genetics Computer Group (GCG) program packages [35] and sequences were submitted to the EMBL Nucleotide Sequence Database under the accession numbers AJ003031-AJ003038. Frankia genus- and group-specific oligonucleotide primers were designed based on sequence differences (Fig. 1).

\subsection{Rep-PCR fingerprinting using $B O X$ primer}

Rep-PCR from DNA of the isolates and reference strains of Frankia (EAN, ACN, WgCc1.17) was performed in a total volume of $25 \mu \mathrm{l}$ containing approximately $100 \mathrm{ng}$ of template DNA, $100 \mathrm{pmol}$ of BOXA1R primer [36], $1.25 \mathrm{mM}$ of dNTPs, 2 units of DNA polymerase (Dynazyme, Finnzymes, Espoo, Finland) in a reaction buffer with $10 \%$ (v/v) dimethyl sulfoxide, $4 \mu \mathrm{g}$ bovine serum albumin (BSA) and 5 $\mathrm{mM} \mathrm{MgCl}_{2}$ [37,36]. PCR amplifications were performed in an automated thermal cycler (Programable Thermal Controller, MJ Research Inc.) with an initial denaturation $\left(95^{\circ} \mathrm{C}, 7 \mathrm{~min}\right)$ followed by 30 cycles of denaturation $\left(95^{\circ} \mathrm{C}, 30 \mathrm{~s}\right)$, annealing $\left(52^{\circ} \mathrm{C}, 1 \mathrm{~min}\right)$, and extension $\left(65^{\circ} \mathrm{C}, 8 \mathrm{~min}\right)$ with a single final extension $\left(65^{\circ} \mathrm{C}, 16 \mathrm{~min}\right)$ [36]. PCR products $(10 \mu \mathrm{l})$ were analyzed by gel electrophoresis on a $1 \%(\mathrm{w} / \mathrm{v})$ Synergel (Diversified Biotech, Boston, MA) agarose gel in TAE buffer (40 mM Tris, 20 $\mathrm{mM}$ acetic acid, $1 \mathrm{mM}$ EDTA, $\mathrm{pH} 8.0$ ) at $5 \mathrm{~V}$ $\mathrm{cm}^{-1}$. For analysis and dendrogram construction the gels were scanned with a CCD camera and the 
obtained images were analyzed using BioImage processing apparatus (Millipore). The patterns were compared using information about apparent molecular masses of the bands and band spacing. The results were used to construct an UPGMA (unweighed pair group method with arithmetic average) dendrogram without using a correction [38].

\subsection{In situ hybridization}

Pure cultures of the isolates and reference strains of Frankia (AgB1.9, ArI3, Ag45/Mut15, AvN17s) [25] (Fig. 1) as well as root nodules of $A$. incana capture plants (60 lobes in total consisting of 20 lobes induced by each soil) were fixed in $4 \%(\mathrm{w} / \mathrm{v})$ paraformaldehyde in phosphate-buffered saline (PBS) [39] at $4^{\circ} \mathrm{C}$ for $16 \mathrm{~h}$. Fixed cells and nodule lobes were subsequently washed in PBS and lobes ground in a mortar. Pure cultures and lobe homogenates were stored in 50\% (v/v) ethanol in PBS at $-20^{\circ} \mathrm{C}$ [39]. Samples $(2 \mu \mathrm{l})$ were spotted onto gelatin-coated slides $(0.1 \% \quad(\mathrm{w} / \mathrm{v})$ gelatin, $0.01 \%$ $\left.\mathrm{KCr}\left(\mathrm{SO}_{4}\right)_{2}\right)$ and allowed to air-dry. After dehydration in 50, 80 and $96 \%(\mathrm{v} / \mathrm{v})$ ethanol for $3 \mathrm{~min}$ each, the preparations were subjected to a pretreatment with lysozyme (Fluka, $1 \mathrm{mg}$ corresponding to $32320 \mathrm{U}$ dissolved in $1 \mathrm{ml}$ of $100 \mathrm{mM}$ Tris- $\mathrm{HCl}$, $\mathrm{pH} 7.5,5 \mathrm{mM}$ EDTA) at $37^{\circ} \mathrm{C}$ for $30 \mathrm{~min}$. Afterwards, the samples were rinsed with distilled water and dehydrated as described above.

Oligonucleotide probes targeting specific sequences on the 23S rRNA insertion of Frankia strains belonging to different groups of the Alnus host infection group (Fig. 1; 23Mut(II), 23AvC, and 23AvN) were synthesized with a primary amino group at the 5 ' end (C6-TFA, MWG). The fluorescent dye Cy3 (Amersham) was covalently bound to the amino group of the oligonucleotide. The dye-oligonucleotide conjugate $(1: 1)$ was purified from unreacted components and stored at $-20^{\circ} \mathrm{C}$ in distilled water at a concentration of $25 \mathrm{ng} \mathrm{ml}^{-1}$ [40]. This solution was amended with the DNA specific dye 4'6-diamidino-2-phenylindole (DAPI) (Sigma) to a final concentration of $10 \mu \mathrm{g} \mathrm{ml}^{-1}$.

Hybridizations were performed in $9 \mu \mathrm{l}$ of hybridization buffer $(900 \mathrm{mM} \mathrm{NaCl}, 20 \mathrm{mM}$ Tris- $\mathrm{HCl}$, $0.01 \%(\mathrm{v} / \mathrm{v}) \mathrm{SDS}, \mathrm{pH} 7.2)$ in the presence of $30 \%$ (v/v) formamide [41] and $1 \mu 1$ of oligonucleotide probe $(25 \mathrm{ng})$ at $42^{\circ} \mathrm{C}$ for $2 \mathrm{~h}$ [27]. After hybridization, the slides were washed in hybridization buffer without formamide at $42^{\circ} \mathrm{C}$ for $20 \mathrm{~min}$, rinsed with distilled water, and air-dried. Slides were finally mounted with Citifluor solution (Citifluor, Canterbury, UK) and the preparations were examined with a Zeiss Axiophot microscope fitted for epifluorescence with a high-pressure mercury bulb $(50 \mathrm{~W})$ and filter sets 02 (Zeiss; G 365, FT 395, LP 420) and HQ-CY3 (AHF Analysentechnik, Tübingen, Germany; G 535/50, FT 565, BP 610/75).

Specificity of and hybridization conditions for Cy3-labeled oligonucleotide probes $23 \mathrm{AvC}, 23 \mathrm{AvN}$, and 23Mut(II) were controlled on reference Frankia strains as well as on isolates. For the analysis of uncultured Frankia populations in lobe homogenates, permeability of Frankia cells was always tested by hybridization with the bacterial probe Eub338 [40] whereas non-specific binding of probes was investigated by hybridization with the oligonucleotide probe Euk516 [40] targeting 18S rRNA of Eukarya.

\subsection{PCR detection of uncultured Frankia populations}

For detection of uncultured Frankia populations in soils, DNA was amplified using primer combinations with different specificities (Fig. 1). Primers FraV and 23Fra were used to specifically detect members of the genus Frankia, primers FraV and 23B1.9 to detect frankiae of the Alnus host infection group I; primers $\mathrm{AFAr}$ and $23 \mathrm{AvC}$ were used to detect frankiae of the Alnus host infection group III and primer combinations AFMr-23AvN and AFMr-23Mut(II) to detect frankiae belonging to group IV.

PCR was performed in a total volume of $100 \mu \mathrm{l}$ containing approximately $100 \mathrm{ng}$ of template DNA, $100 \mathrm{pmol}$ of each primer, $10 \mathrm{mM}$ of dNTPs, 1 unit of Taq polymerase (Appligene) in a reaction buffer with $0.1 \%$ Triton $100,4 \mu \mathrm{g}$ BSA and $2.5 \mathrm{mM} \mathrm{MgCl}_{2}$. Thirty rounds of temperature cycling (Crocodile II Thermocycler, Appligene) of denaturation at $95^{\circ} \mathrm{C}$, primer annealing at $50^{\circ} \mathrm{C}(\mathrm{FraV}-23 \mathrm{Fra}$ and $\mathrm{FraV}$ 23B1.9), $52^{\circ} \mathrm{C}$ (AFAr-23AvC and AFMr-23Mut(II)) and $55^{\circ} \mathrm{C}(\mathrm{AFMr}-23 \mathrm{AvN})$, and elongation at $70^{\circ} \mathrm{C}$, each for $30 \mathrm{~s}$, were followed by a final 7-min incubation at $70^{\circ} \mathrm{C}$. Amplification products were subsequently identified by Southern hybridization. 


\subsection{Southern hybridization}

For Southern hybridizations, fragments generated by PCR on DNA of isolates and soils were separated by agarose gel electrophoresis $(3 \%(\mathrm{w} / \mathrm{v})$ agarose in TAE buffer) and transferred onto Magnagraph nylon filters with a VacuGene Blotting Unit (Pharmacia). DNA samples were immobilized by UV light transillumination and hybridized according to [42].

Digoxigenin-labeled probes were generated by PCR using primers FraV-23B1.9, AFAr-23AvC, AFMr-23AvN and AFMr-23Mut(II) (Fig. 1) as described above, except that $3.5 \mathrm{mM}$ of dTTP instead of $10 \mathrm{mM}$ and in addition $1 \mu \mathrm{l}$ of digoxigenin-UTP (Boehringer) were used. DNAs isolated from isolates AiBp3, AiPs1, AiPal and AgB1.9 were used as templates. Digoxigenin-labeled probes were heat-denatured and hybridized in the presence of $50 \%(\mathrm{v} / \mathrm{v})$ formamide at $50^{\circ} \mathrm{C}$ for $16 \mathrm{~h}$.

The formation of stable hybrids was shown by binding of an antibody-alkaline phosphatase conjugate (Boehringer) to the digoxigenin reporter molecule. Alkaline phosphatase activity was visualized by exposure to X-ray film (X-Omat AR, Kodak) after incubation of the filter in the chemiluminescent substrate CSPD (disodium 3-(4-methoxyspiro \{1,2-dioxetane-3,2'-(5'-chloro) tricyclo [3,3,1,1] decan\}-4-yl) phenyl phosphate) according to the manufacturer's instructions (Tropix, Catalys, Wallisellen, Switzerland).

\section{Results and discussion}

\subsection{Analysis of the nodulation capacity of the soil samples}

The analysis of the nodulation capacity of soils of birch, pine, or spruce stands by bioassays using seedlings of $A$. incana as capture plants resulted in nodule formation with all soil amounts studied, even with the lowest amount of soil $(1.25 \mathrm{mg})$. The first prenodules were detected 9 days after inoculation. Seedlings growing in bottles that were inoculated with $0.5 \mathrm{~g}$ of soil had over 200 nodules, those inoculated with $0.025 \mathrm{~g}$ about 70 nodules and those with $0.00125 \mathrm{~g}$ about five nodules, regardless of the origin of the soil. Based on recalculation from nodule num- bers obtained after inoculation with $0.025 \mathrm{~g}$ of soil, the mean nodulation capacity of the soil from the birch stand was $3160 \pm 7$ nodulation units $\mathrm{g}^{-1}$ soil, that of the soil from the pine stand $2267 \pm 13$ nodulation units $\mathrm{g}^{-1}$ soil, and that of the soil from the spruce stand $2747 \pm 6$ nodulation units $\mathrm{g}^{-1}$ soil.

The nodulation capacities of all three soils are comparable to those found in soils of different locations which range from 0 to up to 4600 nodulation units $\mathrm{g}^{-1}$ soil (as reviewed in [11]), but were higher than those obtained in another study [16]. In contrast to the published data [16-18] which indicate much smaller nodulation capacities of soil from both the pine stand (0-63 nodulation units $\mathrm{cm}^{-3}$ of soil, assuming a bulk density of $1.0 \mathrm{~g} \mathrm{~cm}^{-3}$ ) and the spruce stand $\left(0-50\right.$ nodulation units $\left.\mathrm{cm}^{-3}\right)$ than of soil from a birch stand (490-6500 nodulation units $\mathrm{cm}^{-3}$ ), nodulation capacities of soils from birch, pine and spruce stands in our study are within the same range. This may be attributed to the high similarity of physico-chemical factors in the soils used in our study as well as to identical seasonal conditions which are both supposed to significantly influence nodulation capacities of soils $[43,16]$.

Nodulation capacities of soils are assumed to be influenced by the vegetation $[43,18,16]$ and by variable compatibilities of different Frankia populations to capture plants $[44,20,45,22]$. Although our studies do not show an obvious impact of the vegetation on the overall nodulation capacity, which is in contrast to earlier studies [18], it may be speculated whether differences in vegetation lead to shifts in the overall composition of Frankia populations in soils or to changes in the nodulation capacity of certain Frankia populations.

\subsection{Analysis of isolates obtained from root nodules}

Six Frankia isolates (AiBp1-6) were obtained from 12 nodules harvested from $A$. incana capture plants inoculated with soil from the birch stand. Four isolates (AiPs1-4) were obtained from six nodules of capture plants inoculated with soil from the pine stand, while only one isolate (AiPa1) could be obtained from six nodules of capture plants inoculated with soil from the spruce stand. Of these, eight isolates (AiBp3-6, AiPs1, 2 and 4, and AiPa1) were selected for further characterization by sequence 


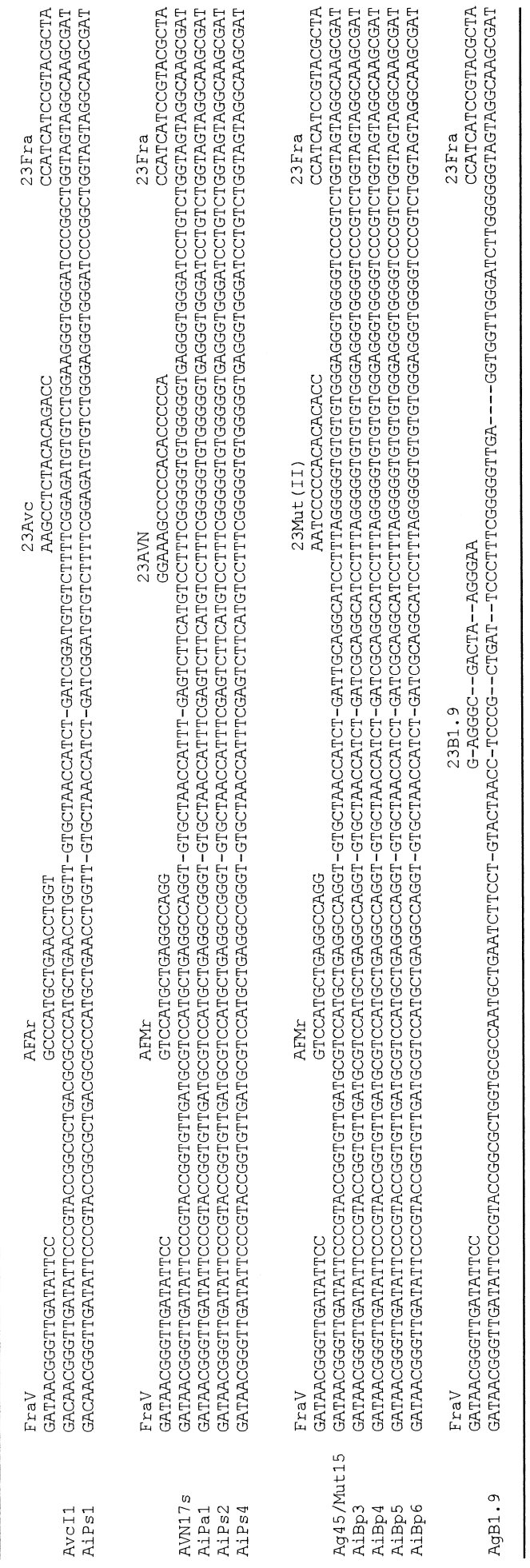

Fig. 1. Alignment of nucleotide sequence fragments containing the insertion in domain III of 23S rRNAs from Frankia strains AvCI1, AvN17s and Ag45/Mut15 [25] and frankiae isolated from nodules of Alnus incana capture plants inoculated with soil obtained from Pinus sylvestris (AiPs), Picea abies (AiPa) and Betula pendula (AiBp) stands [28]. Primer combination FraV and 23Fra was used for genus-specific detection of frankiae by PCR, while primer combinations AFAr and 23AvC, AFMr and 23AvN, and AFMr and 23Mut(II) were used for the detection of groups of Frankia by PCR or nested PCR.

analysis of an actinomycetes-specific insertion in domain III of the 23S rRNA and by rep-PCR.

Based on comparative sequence analysis of PCR amplified 135-bp fragments containing the 23S rRNA insertion of the selected isolates, these isolates could be assigned to two groups of Frankia within the Alnus host infection group according to the classification by [25], namely group IIIa and group IV (Fig. 1). The assignment was partially correlated to the origin of the soil used as inoculum. Sequences of isolates obtained from nodules formed after inoculation with soil from the birch stand (AiBp3-6) were identical and showed only one difference to that of Frankia strain Ag45/Mut15 which was used as the reference strain for the Alnus host infection group IV [25]. Sequences of these isolates revealed 21 differences to the sequence of isolate AiPs1 obtained from nodules formed after inoculation with soil from the pine stand. The sequence of isolate AiPs1 showed one difference compared to the sequence of Frankia strain AvcI1 belonging to the Alnus host infection group IIIa (Fig. 1) [25]. Sequences of isolates AiPs2 and AiPs4, obtained from nodules after inoculation with soil of the same pine stand, as well as that of isolate AiPal, obtained from nodules formed after inoculation with soil from the spruce stand, were identical but showed 27 differences compared to the sequence of isolate AiPs1 and 18 differences compared to those of isolates AiBp3 to AiBp6. Isolates AiPs2, AiPs4 and AiPal showed two differences to the sequence of Frankia strain AvN17s which was assigned to the Alnus host infection group IV [25].

Due to the already observed sequence differences of strain AvN17s compared to the reference strain of group IV, strain Ag45/Mut15 [25], a reorganization of the Alnus host infection group IV into two groups IVa and IVb with Frankia strains Ag45/Mut15 and 

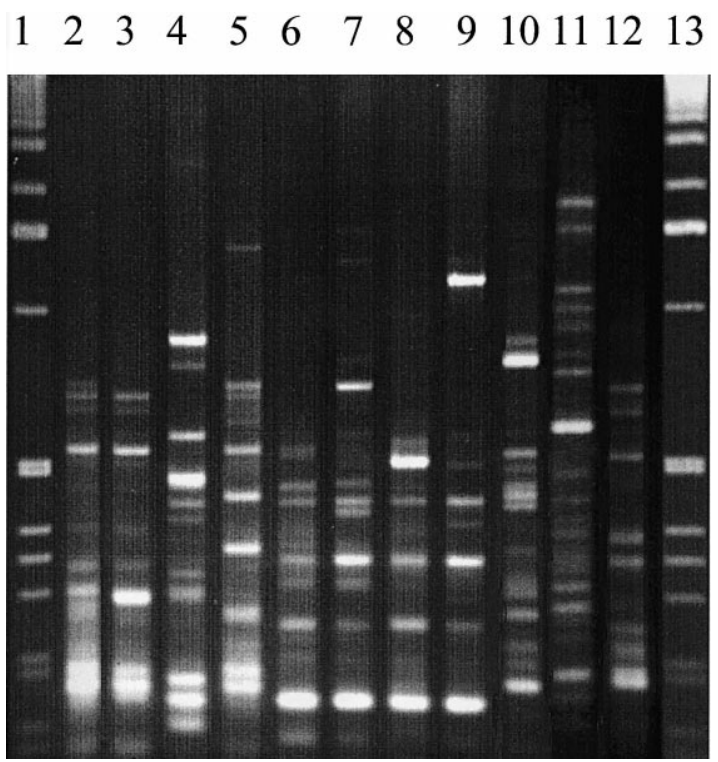

Fig. 2. Rep-PCR fingerprint patterns of genomic DNA from isolates and Frankia strains using BOXA1R primer. Lanes 1 and 13, 1-kb marker; 2, isolates AiPs4; 3, AiPs2; 4, AiPs1; 5, AiPa1; 6, AiBp6; 7, AiBp5; 8, AiBp4; 9, AiBp3; 10, Frankia strain $\mathrm{WgCc1.17}$; 11, Frankia strain ACNs; 12, Frankia strain EAN.

AvN17s as reference strains for the respective groups is justified. This reorganization results in the assignment of all isolates into three distinct groups of the
Alnus host infection groups, namely groups IIIa, IVa and IVb. This assignment was confirmed by the analysis of genomic fingerprints of the eight isolates generated by rep-PCR. On average, 14 bands representing a unique fingerprint for each strain were generated (Fig. 2). Patterns of isolates AiBp3-6 were very similar to each other but distinct from fingerprints of isolates AiPs1, AiPs2, AiPs4 and AiPa1. Fingerprints from isolate AiPal and isolates AiPs2 and AiPs4 again were similar to each other but distinct from others (Fig. 2). An UPGMA dendrogram of the fingerprint patterns shows that the isolates and reference strains could be clustered into five groups (Fig. 3): group A includes isolates AiPa1, AiPs2 and AiPs4, group B includes isolates AiBp3 to AiBp6 and reference strain EAN, group $\mathrm{C}$ includes isolate AiPs1, group D reference strain $\mathrm{WgCc} 1.17$ and group E reference strain ACNs.

Considering that rep-PCR fingerprints may reflect strain-specific genomic structure [36], these results show that each isolate represents a unique strain. Isolates with similar fingerprints can be assigned to the same group within the Alnus host infection group. A comparably good correlation between degrees of relatedness determined by comparative RNA sequence analysis and fingerprinting has been shown for 16S rRNA analysis and rep-PCR fingerprinting of Frankia strains [37]. Partial 23S rRNA

\section{Similarity (\%)}
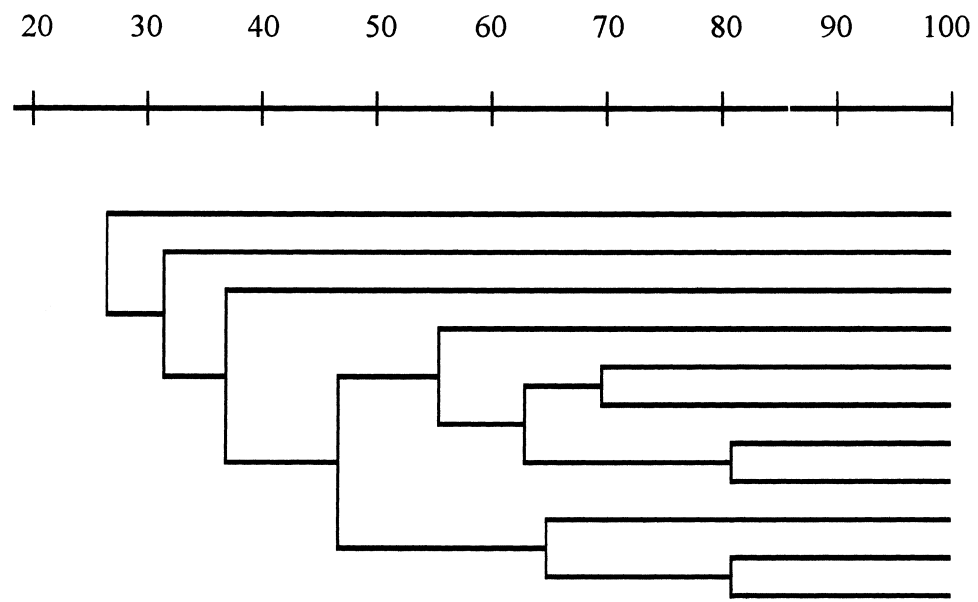

$\begin{array}{ll}\text { ACNs } & \text { E } \\ \text { AiPs1 } & \text { C } \\ \text { WgCc1.17 D } \\ \text { EAN } & \text { B } \\ \text { AiBp3 } & \text { B } \\ \text { AiBp5 } & \text { B } \\ \text { AiBp4 } & \text { B } \\ \text { AiBp6 } & \text { B } \\ \text { AiPa1 } & \text { A } \\ \text { AiPs2 } & \text { A } \\ \text { AiPs4 } & \text { A }\end{array}$

Fig. 3. An UPGMA dendrogram obtained from DNA fingerprint patterns of isolates and Frankia strains. 
sequence analysis as well as rep-PCR fingerprints show that different Frankia strains belonging to the same group within the Alnus host infection group inhabit the same soil and are infective on A. incana. It also suggests that depending on the soil, different Frankia populations may nodulate the capture plants. This assumption, however, is only based on the analysis of a limited number of isolates obtained from nodules. Because isolation procedures can be extremely selective, a final proof on whether the isolates represent the total Frankia populations in nodules must include a comparison of isolates with uncultured Frankia populations in root nodules.

\subsection{Analysis of uncultured Frankia populations in root nodules}

Uncultured Frankia populations in root nodules were analyzed by in situ hybridization. In situ hybridization with the bacterial probe Eub338 on lobe homogenates resulted in intensive hybridization signals on vesicles in all samples. This demonstrated the presence of sufficient permeability of the vesicles and sufficient amounts of target sequences per cell. Frankia populations in lobes induced after inoculation with soil from the birch stand exclusively hybridized with probe $23 \mathrm{Mut}(\mathrm{II})$ thereby confirming the presence of frankiae of the Alnus host infection group IVa. No hybridization signals were obtained with probe $23 \mathrm{AvN}$ targeting Frankia of group IVb or with probe $23 \mathrm{AvC}$ targeting Frankia of group III. The analysis of Frankia populations in lobes induced after inoculation with soil from the pine stand confirmed the presence of frankiae of the Alnus host infection groups III and IVb. Frankia populations in five lobes hybridized with probe $23 \mathrm{AvC}$ and in eight lobes with probe $23 \mathrm{AvN}$. No hybridization signals were obtained with probe $23 \mathrm{Mut}(\mathrm{II})$. In lobes induced after inoculation with soil from the spruce stand, Frankia populations in seven lobes hybridized with probe $23 \mathrm{Mut}(\mathrm{II})$ and in five lobes with probe $23 \mathrm{AvN}$. Here, no hybridization signals were obtained with probe $23 \mathrm{AvC}$. A comparative analysis of the latter samples with isolates, however, was largely hampered by the availability of only one spruce soil isolate, AiPa1.

These results demonstrate that the isolates represent a significant portion of the Frankia population in root nodules of the capture plants after inoculation with soil from the birch, pine or spruce stands. However, only isolates obtained from nodules on capture plants after inoculation with soil from the birch stand seem to represent the total Frankia population in root nodules. In nodules induced after inoculation with soil from the pine or spruce stand, Frankia populations could only be identified with specific probes in 13 out of 20 lobes and 12 out of 20 lobes, respectively, though all lobes contained vesicles which could be hybridized with the bacterial probe Eub338. Therefore, nodules from both stands also contained Frankia populations which had not been isolated and which could not be identified.

Neither in situ hybridization nor DAPI staining could detect spores in any nodule lobe, indicating that all nodules were of the spore $(-)$ type. This observation is in accordance with previous results which showed that soil from sites devoid of host plants did not induce nodules of the spore (+) type [18].

Because the design of the group-specific oligonucleotide probes was based on only a limited amount of pure cultures [25], further studies on the diversity of Frankia populations in nodules may result in the discovery of new Frankia populations. Here, PCRbased techniques can help to obtain sequence information from uncultured Frankia populations which can eventually be used to design specific probes. Nevertheless, these results demonstrate that different Frankia populations form nodules on the capture plants after inoculation with soil from the birch, pine or spruce stands. However, it is not known whether these populations in nodules represent the total Frankia population in these soils or only a fraction of physiologically active, infective frankiae.

\subsection{Analysis of uncultured Frankia populations in soils}

Uncultured Frankia populations in soil were analyzed by PCR on DNA extracted from soil of the birch, pine and spruce stand. However, no amplification products were obtained with specific primer combinations FraV and 23B1.9, AFAr and 23AvC, AFMr and 23AvN or AFMr and 23Mut(II). To increase the sensitivity of detection, a nested PCR using these primer combinations and a portion of PCR 


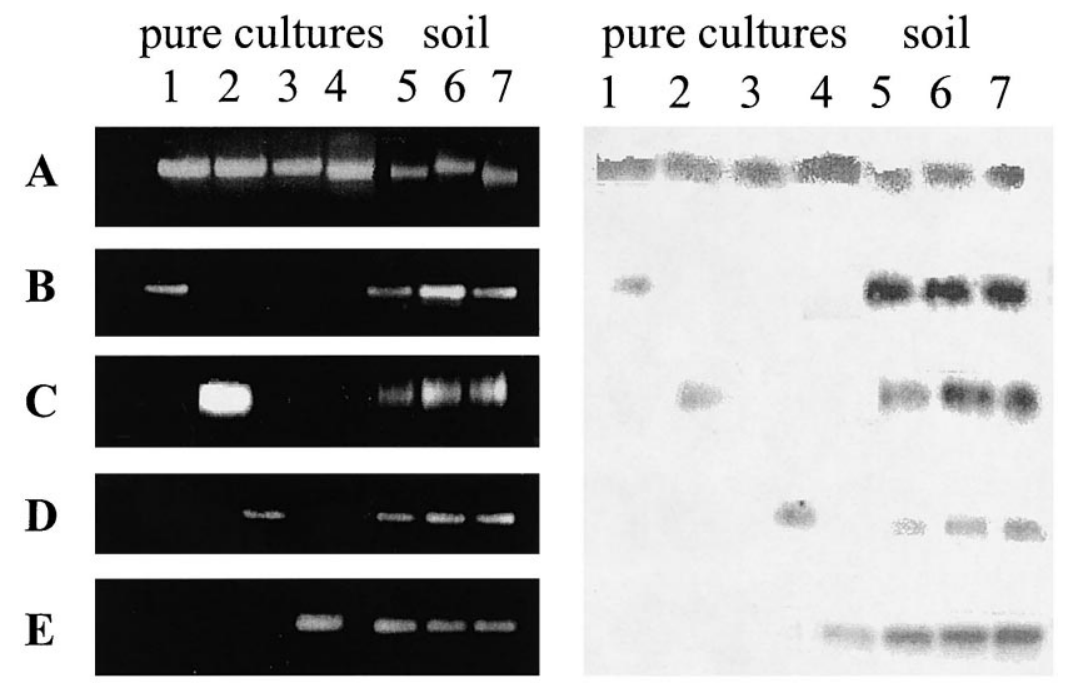

Fig. 4. Gel electrophoretic analysis of PCR products detecting all members of the genus Frankia (A) or specific subgroups of the Alnus host infection group (groups IIIa (B), IVb (C), IVa (D) and I (E), respectively) using DNA of representative isolates (lanes 1-4, respectively) or of soils from birch, spruce or pine stands (lanes 5-7). The right panel shows the concomitant figure after Southern hybridization with digoxigenin-labeled PCR products generated to specifically detect the representative isolates in the group-specific PCRs only (B-E). Hybridization on PCR products detecting all members of the genus (A) was carried out using a combination of all probes.

products obtained after genus-specific amplification with primers FraV and 23Fra as template was necessary. PCR products of approximately $135 \mathrm{bp}$ were obtained on all samples when DNA isolated from reference strains as well as from soil was used as template for a genus-specific amplification (Fig. 4A). Southern hybridization with a combination of probes targeting Frankia strains AiPs1, AiPs4, AiBp3 and AgB1.9 showed signals on all PCR products, demonstrating the presence of homologous sequences (Fig. 4A).

A nested PCR with specific primer combinations resulted in amplification products of approximately $65 \mathrm{bp}$ on DNA extracted from soil of the birch, pine and spruce stands, as well as on DNA obtained from pure cultures of the respective target groups (Fig. 4B-E). The presence of homologous sequences was confirmed by hybridization with probes targeting representative strains of the respective target groups (Fig. 4B-E).

These PCR-based results indicate a similar diversity of the total Frankia population in soils of the birch, pine and spruce stands. However, a PCRbased analysis does not necessarily reflect the abundances in the original sample [46]. Therefore, the size of the Frankia populations detected may differ sig- nificantly. Furthermore, the analysis only focused on a small number of target groups and may therefore be incomplete. This assumption is supported by the discovery of Frankia populations in nodules of the capture plants after inoculation with soil from the pine and spruce stands which could not be identified.

Based on the analysis of Frankia populations in nodules and soils, however, it must be assumed that Frankia populations in nodules of the capture plants represent the fraction of physiologically active, infective frankiae in the test soils rather than the total Frankia population. This assumption is supported by studies in which only one population of Frankia was detected in nodules of the host plant at the respective site by in situ hybridization, though different Frankia populations were detected in soil by PCR [27]. Moreover, comparative analysis of nodulation units with genomic units of soils determined by the PCR-MPN technique using nested PCR $[11,43]$ indicated that only a small portion of the total population of Frankia was able to nodulate. It was suggested that the nodulation capacity of a soil was controlled largely by the physiological status of the Frankia populations rather than by the total population size [43]. 


\section{Acknowledgments}

We gratefully acknowledge Aino Smolander (Finnish Forest Research Institute) for sharing her expertise on Frankia research and for providing the soil samples. We also thank Jarkko Hantula for assistance in automated clustering analysis and Lars Paulin for the sequence analysis. This work was funded by the Academy of Finland, the Helsinki University Graduate School Programme and grants from the Finnish Cultural Foundation, the Swiss National Science Foundation (Priority Program Biotechnology) and the Swiss Federal Office of Environment, Forests and Landscape (BUWAL).

\section{References}

[1] Baker, D. (1988) Opportunities for autecological studies of Frankia, a symbiotic actinomycete. In: Biology of Actinomycetes '88 (Okami, Y., Beppu, T. and Ogawara, H., Eds.), pp. 271-276. Scientific Societies Press, Tokyo, Japan.

[2] Schwintzer, C.R. and Tjepkema, J.D. (1990) The Biology of Frankia and Actinorhizal Plants. Academic Press, San Diego, CA.

[3] Baker, D. and O'Keefe, D. (1984) A modified sucrose fractionation procedure for the isolation of frankiae from actinorhizal root nodules and soil samples. Plant Soil 78, 23-28.

[4] Baker, D. (1990) Methods for the isolation, culture and characterization of the Frankiaceae: Soil actinomycetes and symbionts of actinorhizal plants. In: Isolation of Biotechnological Organisms from Nature (Labeda, H.D., Ed.), pp. 213-236. McGraw Hill, New York.

[5] Stowers, M.D. (1987) Collection, isolation, cultivation and maintenance of Frankia. In: Symbiotic Nitrogen Fixation Technology (Elkan, G.H., Ed.), pp. 29-53. Marcel Dekker, New York.

[6] Rosbrook, P.A., Burggraaf, A.J.P. and Reddell, P. (1989) A comparison of two methods and different media for isolating Frankia from Casuarina root nodules. Plant Soil 120, 187-193.

[7] St-Laurent, L. and Lalonde, M. (1987) Isolation and characterization of Frankia strains isolated from Myrica gale. Can. J. Bot. 65, 1356-1363.

[8] Quispel, A. (1954) Symbiotic nitrogen fixation in non-leguminous plants. II. The influence of the inoculation density and external factors on the nodulation of Alnus glutinosa and its importance to our understanding of the mechanism of infection. Acta Bot. Neerl. 3, 512-532.

[9] Hilger, A.B., Tanaka, Y. and Myrold, D.D. (1991) Inoculation of fumigated nursery soil increases nodulation and yield of bare-root red alder (Alnus rubra Bong.). New Forests 5, 3542.

[10] Huss-Danell, K. and Myrold, D.D. (1994) Intrageneric variation in nodulation of Alnus - consequences for quantifying
Frankia nodulation units in soil. Soil Biol. Biochem. 26, 525-531.

[11] Myrold, D.D., Hilger, A.B., Huss-Danell, K. and Martin, K.J. (1994) Use of molecular methods to enumerate Frankia in soil. In: Beyond the Biomass (Ritz, K., Dighton, J. and Giller, K.E., Eds.), pp. 127-136. John Wiley and Sons, Chichester.

[12] Arveby, A.S. and Huss-Danell, K. (1988) Presence and dispersal of infective Frankia in peat and meadow soils in Sweden. Biol. Fertil. Soils 6, 39-44.

[13] Burleigh, S.H. and Dawson, J.O. (1994) Occurrence of Myrica-nodulating Frankia in hawaiian volcanic soils. Plant Soil 164, 283-289.

[14] Huss-Danell, K. and Frej, A.-K. (1986) Distribution of Frank$i a$ in soils from forest and afforestation sites in northern Sweden. Plant Soil 90, 407-417.

[15] Smolander, A. and Sarsa, M.-L. (1990) Frankia strains of soil under Betula pendula: Behaviour in soil and pure culture. Plant Soil 122, 129-136.

[16] Smolander, A. and Sundman, V. (1987) Frankia in acid soils of forests devoid of actinorhizal plants. Physiol. Plant 70, 297 303.

[17] Smolander, A., van Dijk, C. and Sundman, V. (1988) Survival of Frankia strains introduced into soil. Plant Soil 106, 65-72.

[18] Smolander, A. (1990) Frankia populations in soils under different tree species-with special emphasis on soils under Betula pendula. Plant Soil 121, 1-10.

[19] Baker, D. (1987) Relationship among pure cultured strains of Frankia based on host specificity. Physiol. Plant 70, 245248.

[20] Hahn, D., Starrenburg, M.J.C. and Akkermans, A.D.L. (1988) Variable compatibility of cloned Alnus glutinosa ecotypes against ineffective Frankia strains. Plant Soil 107, 233 243

[21] Hahn, D., Starrenburg, M.J.C. and Akkermans, A.D.L. (1990) Growth increment of Alnus glutinosa upon dual inoculation with effective and ineffective Frankia strains. Plant Soil $122,121-127$.

[22] Van Dijk, C., Sluimer, A. and Weber, A. (1988) Host range differentiation of spore ${ }^{+}$and spore ${ }^{-}$strain types of Frankia in stands of Alnus glutinosa and Alnus incana in Finland. Physiol. Plant 72, 349-358.

[23] Weber, A., Nurmiaho-Lassila, E.L. and Sundman, V. (1987) Features of the intrageneric Alnus-Frankia specificity. Physiol. Plant 70, 289-296.

[24] Weber, A., Sarsa, M.L. and Sundman, V. (1989) Frankia-Alnus incana symbiosis: Effect of endophyte on nitrogen fixation and biomass production. Plant Soil 120, 291-297.

[25] Hönerlage, W., Hahn, D., Zepp, K., Zeyer, J. and Normand, P. (1994) A hypervariable 23S rRNA region provides a discriminating target for specific characterization of uncultured and cultured Frankia. Syst. Appl. Microbiol. 17, 433-443.

[26] Zepp, K., Hahn, D. and Zeyer, J. (1997) Evaluation of a 23S rRNA insertion as target for the analysis of uncultured Frankia populations in root nodules of alders by whole cell hybridization. Syst. Appl. Microbiol. 20, 124-132.

[27] Zepp, K., Hahn, D. and Zeyer, J. (1997) In situ analysis of introduced and indigenous Frankia populations in soil and 
root nodules obtained on Alnus glutinosa. Soil Biol. Biochem. 29, 1595-1600.

[28] Priha, O. and Smolander, A. (1997) Microbial biomass and activity in soil and litter under Pinus sylvestris, Picea abies and Betula pendula at originally similar field afforestation sites. Biol. Fertil. Soils 24, 45-51.

[29] Normand, P. and Lalonde, M. (1982) Evaluation of Frankia strains isolated from provenances of two Alnus species. Can. J. Microbiol. 28, 1133-1142.

[30] Weber, A., Smolander, A., Nurmiaho-Lassila, E.-L. and Sundman, V. (1988) Isolation and characterization of Frankia strains from Alnus incana and Alnus glutinosa in Finland. Symbiosis 6, 97-116.

[31] Sambrook, J., Fritsch, E.F. and Maniatis, T. (1989) Molecular Cloning: A Laboratory Manual. Cold Spring Harbor Laboratory Press, Cold Spring Harbor, NY.

[32] Hönerlage, W., Hahn, D. and Zeyer, J. (1995) Detection of mRNA of nprM in Bacillus megaterium ATCC 14581 grown in soil by whole cell hybridization. Arch. Microbiol. 163, 235241.

[33] Tsai, Y.-L. and Olson, B. (1992) Rapid method for separation of bacterial DNA from humic substances in sediments for polymerase chain reaction. Appl. Environ. Microbiol. 58, 2292-2295.

[34] Roller, C., Ludwig, W. and Schleifer, K.-H. (1992) Grampositive bacteria with a high DNA $\mathrm{G}+\mathrm{C}$ content are characterized by a common insertion within their $23 \mathrm{~S}$ rRNA genes. J. Gen. Microbiol. 138, 1167-1175.

[35] Devereux, J., Haeberli, P. and Smithies, O. (1984) A comprehensive set of sequence analysis programs for the VAX. Nucleic Acids Res. 12, 387-395.

[36] Versalovic, J., Schneider, M., de Bruijn, F.J. and Lupski, J.R. (1994) Genomic fingerprinting of bacteria using repetitive sequence-based polymerase chain reaction. Methods Mol. Cell. Biol. 5, 25-40.
[37] Murry, M.A., Zhang, D., Schneider, M. and de Bruijn, F.J. (1995) Use of repetitive sequences and the polymerase chain reaction (rep-PCR) to fingerprint the genomes of Frankia isolates. Symbiosis 19, 223-240.

[38] Sneath, P.H.A. and Sokal, R.R. (1973) Numerical Taxonomy: The Principles and Practice of Numerical Classification, pp. 394 400. Freeman, San Francisco, CA.

[39] Hahn, D., Amann, R.I. and Zeyer, J. (1993) Whole-cell hybridization of Frankia strains with fluorescence- or digoxigenin-labeled, 16S rRNA-targeted oligonucleotide probes. Appl. Environ. Microbiol. 59, 1709-1716.

[40] Amann, R.I., Binder, B.J., Olsen, R.J., Chisholm, S.W., Devereux, R. and Stahl, D.A. (1990) Combination of 16S rRNAtargeted oligonucleotide probes with flow cytometry for analyzing mixed microbial populations. Appl. Environ. Microbiol. 56, 1919-1925.

[41] Manz, W., Amann, R.I., Ludwig, W., Wagner, M. and Schleifer, K.-H. (1992) Phylogenetic oligodeoxynucleotide probes for the major subclasses of proteobacteria: Problems and solutions. Syst. Appl. Microbiol. 15, 593-600.

[42] Church, G.M. and Gilbert, W. (1984) Genomic sequencing. Proc. Natl. Acad. Sci. USA. 81, 1991-1995.

[43] Myrold, D.D. and Huss-Danell, K. (1994) Population dynamics of Alnus-infective Frankia in a forest soil with and without host trees. Soil Biol. Biochem. 26, 533-540.

[44] Weber, A. (1986) Distribution of spore-positive and sporenegative nodules in stands of Alnus glutinosa and Alnus incana in Finland. Plant Soil 96, 205-213.

[45] Van Dijk, C. and Sluimer, A. (1994) Resistance to an ineffective Frankia strain type in Alnus glutinosa (L.) Gaertn. New Phytol. 128, 497-504.

[46] Suzuki, M.T. and Giovannoni, S.J. (1996) Bias caused by template annealing in the amplification of mixtures of $16 \mathrm{~S}$ rRNA genes by PCR. Appl. Environ. Microbiol. 62, 625630 . 